Arch. Histol. Cytol., Vol. 64, No. 2 (2001)

p. $149-158$

\title{
Development of Astrocytes in the Mouse Hippocampus as Tracked by Tenascin-C Gene Expression*
}

\author{
Shigeki YUASA \\ Laboratory of Neural Information, National Institute for Physiological Sciences, Okazaki, Japan \\ Received September 25, 2000; revised January 18, 2001
}

Summary. Tenascin-C (TN-C) is an astroglia-derived extracellular matrix protein that has been shown to be an early marker for astroglial precursors in the embry onic mouse brain. This study examined astroglial generation, migration, and differentiation in the developing mouse hippocampus by in situ hybridization histochemistry for TN-C mRNA. Special reference was given to the difference in the mode of astroglial development between the two cortical structures of the hippocampus: the dentate gyrus and Ammon's horn. TN-C-positive cells were found in the ventricular germinative zone of the hippocampus as early as the 15 th gestational day, and the labeled cells in the zone apposed to the fimbria migrated tangentially through the subpial area towards the forming dentate gyrus. The TN-C-positive cells aligned in the dentate gyrus exhibited the characteristic morphology of unipolar astrocytes as revealed by double labeling with glial fibrillary acidic protein (GFAP)-immunohistochemistry. On the other hand, the TN-C-positive cells ranging over a wide area of the ventricular germinative zone facing the forming Ammon's horn migrated radially towards the cortex, with most of them aligned in the Ammon's horn exhibiting a GFAP-positive stellate morphology. The onset of migration towards the dentate gyrus was two days earlier than that towards the Ammon's horn. TN-C-positive cells in both cortical structures exhibited a DNA-replicating activity after settlement in the early postnatal stage and were considered to further generate astrocytes. On the other hand, TN-C-positive cells with DNA-replicating activity were also found in the subpial migratory stream moving towards the dentate gyrus and were considered to form the subpial matrix for the generation of the dentate astrocytes. Migratory TN-Cpositive cells directed towards both the dentate gyrus and Ammon's horn were apposed to radial glial processes and were believed to be guided by contact with these processes in a manner similar to migratory immature neurons. These findings indicate that TN-C-positive cells for the dentate gyrus and those for the Ammon's horn have different migratory patterns and undergo different morphological differentiations depending on their site of origin at the early stage of astrogliogenesis and corresponding to the different modes of neurogenesis in the two cortical structures.

The early stage of astroglial generation is not well understood because of a lack of specific markers for labeling astroglial precursors prior to the expression of glial fibrillary acidic protein (GFAP), the marker for the late stage of astroglial differentiation. Tenascin-C (TN-C) is an astroglia-derived extracellular matrix protein (BARTSCH et al., 1992; DOERRIES and SCHACHNER, 1994; MiTrovic et al., 1994). Its expression has been shown to be a useful early marker for astroglial precursors, enabling the precursors to be traced from their generation in the ventricular germinative zone to the site of their settlement (YUASA, 2001). Application of this marker to the study of astrogliogenesis in the mouse embryonic cerebrum has revealed that a subset of astroglial precursors are generated directly from the ventricular germinative zone and migrate radially towards the cortex during the period of neurogenesis. In addition, the site of generation and the mode of migration are heterogeneous within the germinative zone of the lateral ventricle (YUASA, 2001).

The generation and migration of immature neurons in various cortical structures of the developing brain have been extensively studied. These heterogeneous processes depend on the site of cell origin and the stratification of cortical structures. The distribution and morphology of astrocytes also vary according to each cortical structure. Most astrocytes

*This study was supported in part by a Grant-in-Aid for Scientific Research provided by the Ministry of Education, Science, Sports and Culture, Japan (No.06671171 and 07279244). 
exhibit a stellate morphology. However, some astrocytes undergo specialized morphological differentiation, such as the astrocytes with unipolar radial processes that are seen in the cerebellar cortex (LUDWIN et al., 1976) and the hippocampal dentate gyrus (LuDWIN et al., 1976; KOSAKA and HAMA, 1986). Thus, astroglial development in different cortical structures is also likely to be heterogeneous.

The hippocampus is composed of two cortical structures, the dentate gyrus and Ammon's horn. The structure and arrangement of the astrocytes in these two cortical structures differ remarkably (KOSAKA and HAMA, 1986). A strong GFAP immunoreactivity appears in the early developmental stage of the hamster dentate gyrus, and the mode of astrocyte generation, migration and differentiation has been studied in this animal model (Sievers et al., 1992). Very little is known about the early stage of astroglial precursors in the developing hippocampus of the mouse because the expression of GFAP occurs at a much later stage as compared with the hamster. Since genetically manipulated mice are extensively used in research on neural development, information on astrogliogenesis in embryonic mice would certainly serve an important purpose.

The present study examined the development of astrocytes in the mouse embryonic and neonatal hippocampus by employing in situ hybridization histochemistry for TN-C mRNA to label the somata of astroglial precursors. Special attention was paid to differences in astroglial development in the dentate gyrus and Ammon's horn, as well as to the mode of generation, migration and differentiation of the characteristic unipolar astrocytes in the dentate gyrus. The interrelations between the migration of TN-Cpositive astroglial precursors and the arrangement of GFAP-positive radial glial processes were also evaluated. Furthermore, the DNA-replicating activity of the $\mathrm{TN}$-C-positive cells was examined in relation to postnatal astrogliogenesis. The relations between astrogliogenesis and neurogenesis in the developing hippocampus are discussed in view of the findings.

\section{MATERIALS AND METHODS}

\section{Animals}

ICR strain mice were used. The day on which a vaginal plug was confirmed was defined as $\mathrm{E} 0$, and the day of birth was defined as $\mathrm{P} 0$.

All the animal experiments were performed in accordance with the Guide for the Care and Use of Laboratory Animals (NIH Publication No. 85-23, revised 1985). The pregnant mice were deeply anesthe- tized by an intraperitoneal administration of Nembutal (Abbott Laboratories, USA), and the embryos were removed by cesarean section. Neonates were anesthetized by hypothermia during the fixation process.

The experiments were repeated in four to six animals for each developmental stage.

\section{In situ hybridization histochemistry}

All the procedures have been previously reported (YUASA, 1996, 2001). Briefly, the cDNA fragment coding the sequence that is unique for $\mathrm{TN}-\mathrm{C}$ among the TN family (SAGA et al., 1991), extending from nucleotide 131 to 706 (GenBank accession number D90343), was digested with $S a c$ I and $B g l$ II and was cloned into the Sac I/Bam H I site of a pBluescript II/KS- vector. The clone was digested with $S m a$ I or Ecl 136 II (Sac I site) and used as a DNA template to synthesize TN-C antisense or sense cRNA probe. TN-C antisense or sense digoxigenin-labeled cRNA probe was prepared employing $\mathrm{T} 7$ or T3-RNA polymerase and a DIG-RNA-labeling kit (BoehringerMannheim, Germany).

Mouse embryos and neonates were fixed by transcardiac perfusion with $4 \%$ paraformaldehyde in a 0.1 $\mathrm{M}$ sodium phosphate buffer (PB, pH 7.4). Cryostat sections (10 $\mu \mathrm{m}$ in thickness) were digested with $0.8 \%$ pepsin (DAKO, USA) in $0.2 \mathrm{M} \mathrm{HCl}$ for 15 minutes at $37^{\circ} \mathrm{C}$. Subsequently, sections were hybridized with the cRNA probe $(1.2 \mu \mathrm{g} / \mathrm{ml})$ in a hybridization solution at $50^{\circ} \mathrm{C}$ for 18 hours. The sections were then rinsed with $2 \times \mathrm{SSC}$ containing $50 \%$ formamide at $50^{\circ} \mathrm{C}$ and treated with RNase A (Sigma, USA; $10 \mu \mathrm{g} / \mathrm{ml}$ ). They were next washed with $2 \times \mathrm{SSC}$ and finally washed with $0.2 \times \mathrm{SSC}$ at $50^{\circ} \mathrm{C}$. The hybridized probe was detected using a DIG-nucleic acid detection kit (Boehringer-Mannheim) and an alkaline phosphataselabeled anti-digoxigenin antibody. The sense cRNA probe was employed as a control, and no signals were detected in the brain.

\section{Double labeling by in situ hybridization and immunohistochemistry}

Following the development of a deep blue color by TN-C in situ hybridization, the sections were rinsed and immunohistochemical staining was performed using a rabbit anti-GFAP antiserum (prediluted, DAKO) as the primary antibody and horseradish peroxidase (HRP)-labeled anti-rabbit IgG (Fab'-fragment, 1: 100 dilution, MBL, Japan) as the secondary antibody. The localization of the antigens was detected with diaminobenzidine (DAB) and $\mathrm{H}_{2} \mathrm{O}_{2}$ by the development of a brown color.

To achieve double labeling with TN-C in situ 


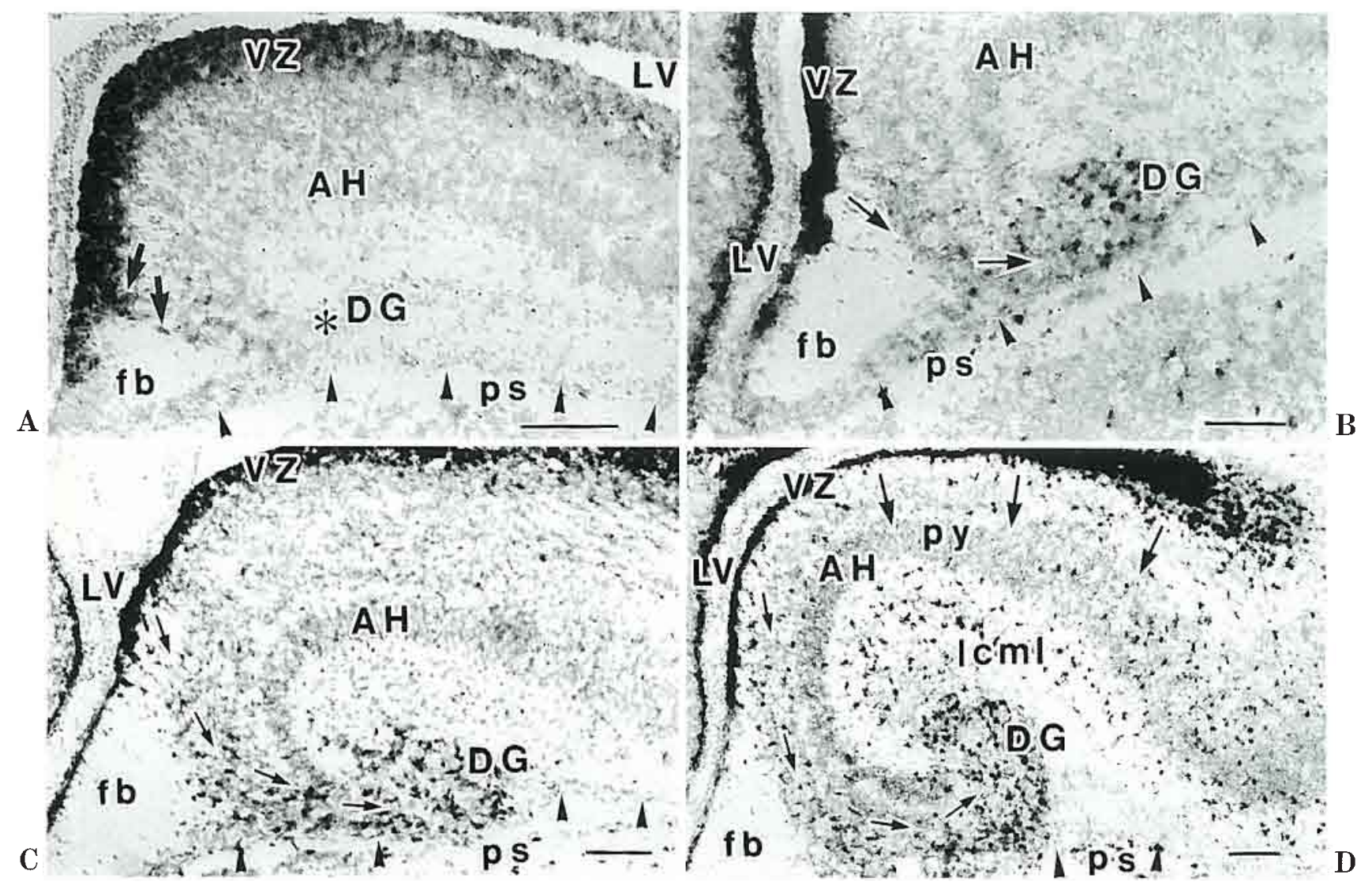

Fig. 1. In situ hybridization histochemistry for TN-C mRNA on parasagittal sections of the embryonic hippocampus. Top is dorsal; left is rostral. A. E15: The labeled cells are aligned in the ventricular germinative zone facing the lateral ventricle. Small numbers of labeled cells (arrows) have migrated from the restricted area apposed to the fimbria. The asterisk indicates the presumptive dentate gyrus. B. E16: The labeled cells have migrated and accumulated in the forming dentate gyrus. The presumptive migratory route is indicated by the arrows. C. E17: The migratory stream (arrows) of labeled cells through the subpial area to the dentate gyrus is evident. D. E18: The migratory stream (small arrows) of labeled cells is still evident, and the labeled cells have begun to form a C-shaped stratification in the dentate gyrus. At this stage, the radial migration of labeled cells from the ventricular germinative zone facing the Ammon's horn has also become visible (large arrows). These labeled cells are settling throughout the entire Ammon's horn. Scale bars: $100 \mu \mathrm{m}$

Abbreviations used in this and other figures: $A H$ Ammon's horn, $D G$ dentate gyrus, $f b$ fimbria, $l \mathrm{~cm} l$ stratum lacunosum/moleculare, $L V$ lateral ventricle, $p s$ pial surface (indicated by the arrowheads in Figure 1), $p y$ pyramidal cell layer, so stratum oriens, sup suprapyramidal blade of dentate gyrus, $S V Z$ subventricular zone, $V Z$ ventricular zone.

hybridization and bromodeoxyuridine (BrdU) immunostaining, BrdU (Sigma, $100 \mathrm{mg} / \mathrm{kg}$ body weight) was administered intraperitoneally to the neonatal mice, and the mice were fixed after $3 \mathrm{~h}$ by transcardiac perfusion with $4 \%$ paraformaldehyde in $\mathrm{PB}$. In situ hybridization for TN-C mRNA was performed on the cryostat sections and the deep blue color was developed. The sections were rinsed and then incubated with $2 \mathrm{M} \mathrm{HCl}$ for $1 \mathrm{~h}$ at room temperature. After rinsing, immunohistochemical staining was performed using mouse anti-BrdU monoclonal antibody $(1: 50$ dilution, Becton-Dickinson, USA) as the primary antibody, and HRP-labeled anti-mouse IgG (Fab'fragment, $1: 100$ dilution, MBL, Japan) as the secondary antibody. The brown color was then developed using $\mathrm{DAB}$ and $\mathrm{H}_{2} \mathrm{O}_{2}$. 

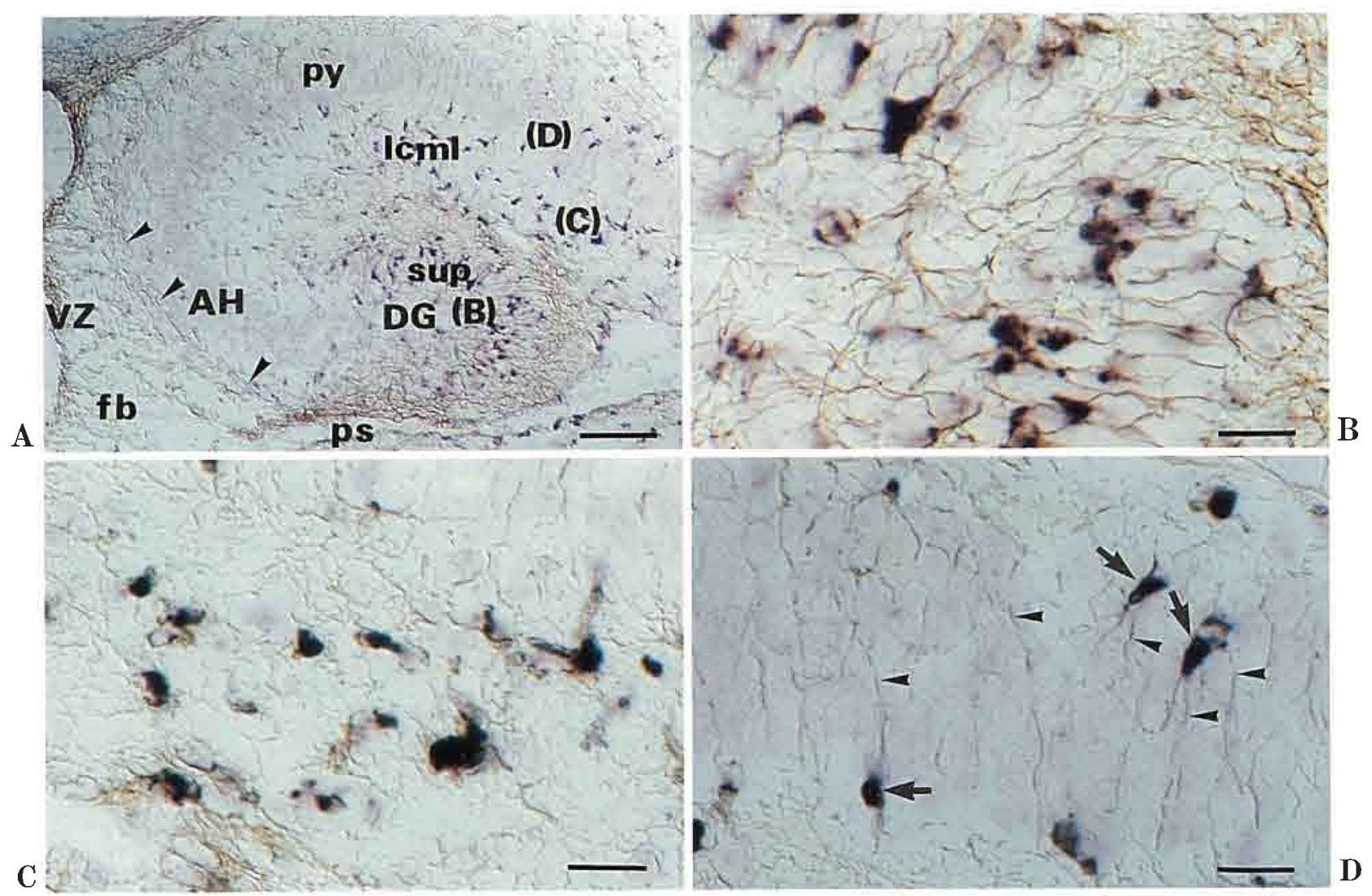

Fig. 2. Transition of TN-C-positive precursors to GFAP-positive astrocytes. Double labeling by TN-C in situ hybridization (deep blue color) and GFAP-immunohistochemistry (brown color) on a hippocampal parasagittal section at P2. Top is dorsal; left is rostral. A. The overall distribution of TN-C-positive cells and GFAP-positive glial arrangement. GFAP-positive radial processes (arrowheads) can be seen. B. Higher magnification of the suprapyramidal blade of the dentate gyrus, as indicated by $(B)$ in Figure 2A. TN-C-positive cells stratified in the granule cell layer have extended GFAP-positive radial processes towards the pia mater and display the morphology of unipolar astrocytes. C. Higher magnification of the stratum lacunosum/moleculare, as indicated by $(C)$ in Figure 2A. TN-C-positive cells have extended GFAP-positive processes and display a stellate morphology. D. Higher magnification of the pyramidal cell layer, as indicated by $(D)$ in Figure 2A. GFAPpositive radial processes (arrowheads) have traversed the pyramidal cell layer, and TN-C-positive cells closely apposed to them (arrows) are occasionally visible. Scale bars: $100 \mu \mathrm{m}(\mathrm{A}), 20 \mu \mathrm{m}(\mathrm{B}-\mathrm{D})$

\section{RESULTS}

\section{Migration and alignment of TN-C-positive cells in the embryonic hippocampus}

At E15 (Fig. 1A), TN-C gene expression in the hippocampal primordium was restricted to the ventricular germinative zone. The intensity of gene expression. was higher in the rostral half of the primordium. The migration of labeled cells began from a limited area of the ventricular germinative zone that was apposed to the fimbria.

At E16 (Fig. 1B), TN-C-positive cells were found in the forming dentate gyrus. Small numbers of labeled cells were also detected in the subpial area on the presumptive migratory route towards the dentate gyrus. The migratory stream of labeled cells thus appeared to have originated from the restricted ventricular germinative zone that was closely apposed to the fimbria and to follow a course through the subpial area towards the dentate gyrus.

At E17 (Fig. 1C), the migratory stream of labeled cells became clearer. The stream evidently originated from the restricted area of the ventricular germinative zone apposed to the fimbria, circumvented the forming Ammon's horn, and was directed towards the dentate gyrus through the subpial area. This migratory route corresponded to the future stratum 

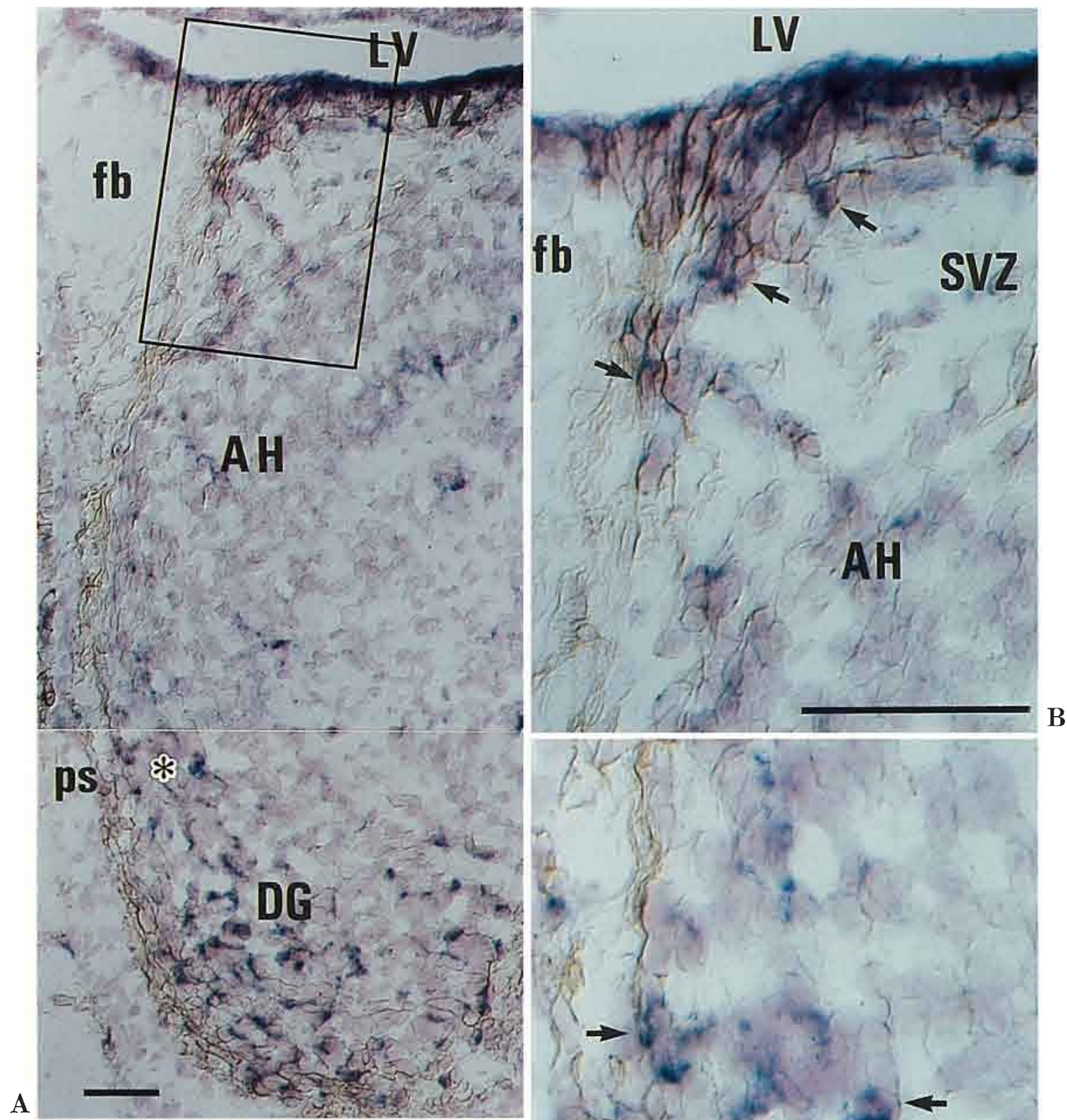

Fig. 3. Correlation between the radial glial arrangement and migratory TN-C-positive cells. Double labeling by TN-C in situ hybridization (deep blue color) and GFAP-immunohistochemistry (brown color) on a hippocampal horizontal section at E17. Top is rostral; left is medial. A. GFAP-positive processes of radial glia originate from the ventricular germinative zone apposed to the fimbria. These processes reach the forming dentate gyrus by extending through the subpial area. TN-C-positive cells follow a migratory course along the radial glial arrangement and accumulate in the dentate gyrus. B. Higher magnification of the boxed area in Figure 3A. GFAP-positive processes have extended from the ventricular germinative zone apposed to the fimbria and have converged to form bundles. TN-C-positive cells in the ventricular germinative zone have migrated into these bundles. The close

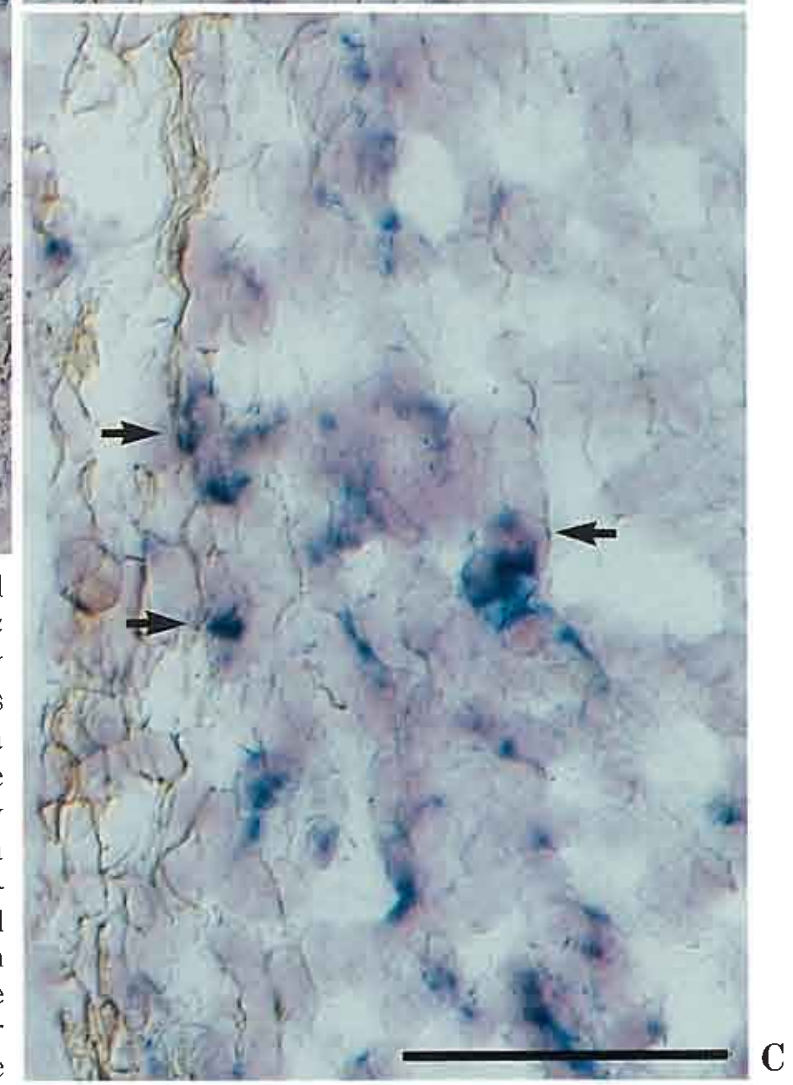
apposition of TN-C-positive cells and radial glial processes is visible (arrows). C. Higher magnification of the subpial area, as indicated by the asterisk in Figure 3A. The apposition of TN-C-positive cells and GFAP-positive processes is evident (arrows). Scale bars: $50 \mu \mathrm{m}$ 

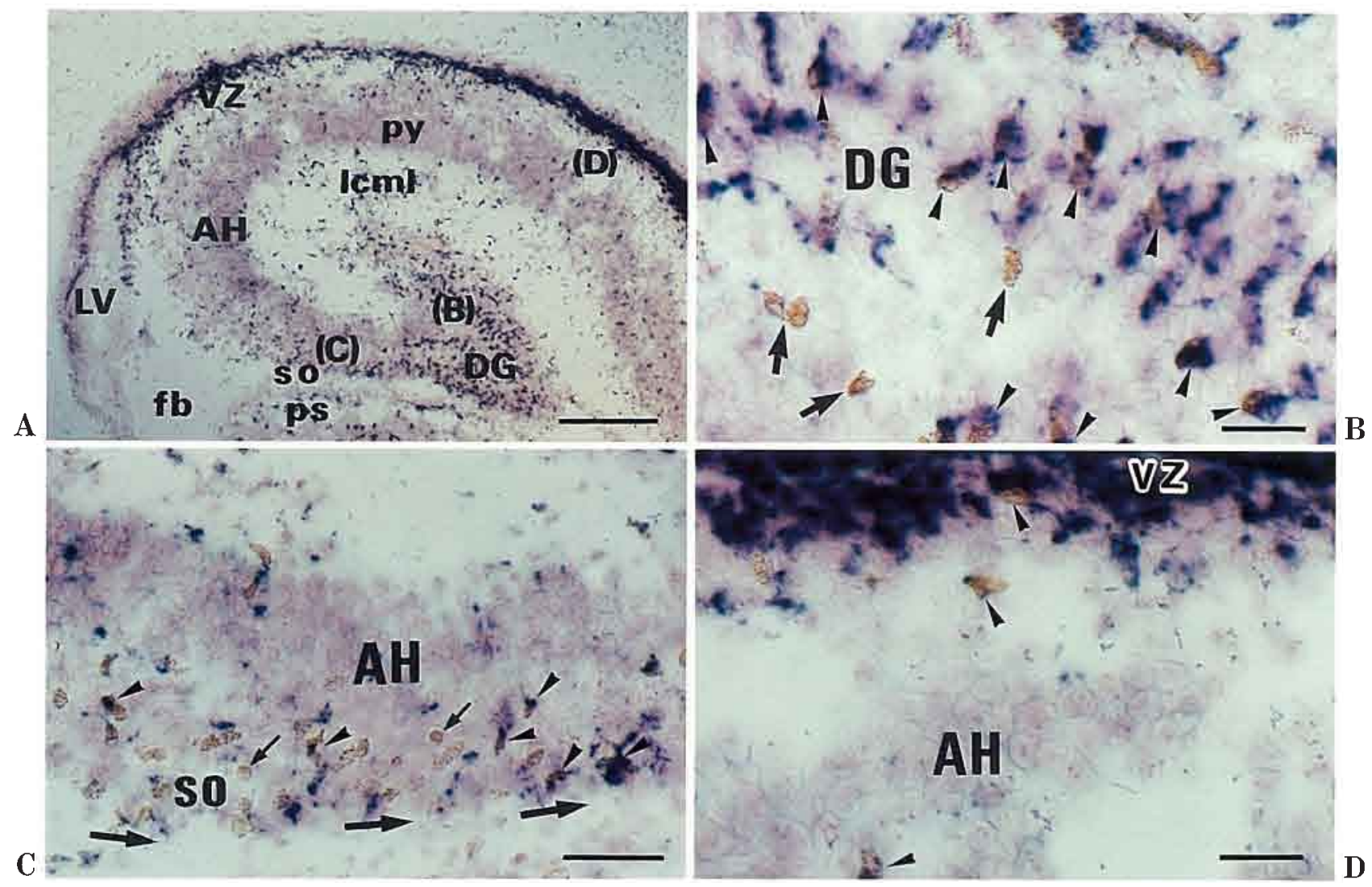

Fig. 4. BrdU uptake and TN-C expression. Double labeling by TN-C in situ hybridization (deep blue color) and BrdU-immunohistochemistry (brown color) on a hippocampal cross section at P0. Top is dorsal; left is medial. A. Overall distribution of the labeled cells. A dense accumulation of labeled cells in the dentate gyrus is visible. The migratory stream towards the dentate gyrus can still be observed in the subpial area. The labeled cells are also distributed in the ventricular germinative zone facing the Ammon's horn and have migrated radially through the pyramidal layer towards the stratum lacunosum/moleculare. B. Higher magnification of the dentate gyrus indicated by $(B)$ in Figure 4A. Double labeled cells (arrowheads) are visible in the granule cell layer. BrdU-positive TN-C-negative cells are occasionally seen (arrows). C. Higher magnification of the subpial area indicated by (C) in Figure 4A. Double labeled cells (arrowheads) are seen following the migratory stream (large arrows). BrdU-positive TN-C-negative cells (small arrows) are present in the stream. D. Higher magnification of the ventricular germinative zone facing the Ammon's horn, as indicated by $(D)$ in Figure 4A. TN-C-positive cells are still densely aligned and small numbers of double labeled cells (arrowheads) can be seen. Scale bars: 200 $\mu \mathrm{m}(\mathrm{A}), 20 \mu \mathrm{m}$ (B and D), $50 \mu \mathrm{m}$ (C)

oriens. The labeled cells accumulated in the dentate gyrus to form a cluster. The radial migration of labeled cells towards the Ammon's horn began from a wide area of the ventricular germinative zone facing the Ammon's horn.

At E18 (Fig. 1D), the migratory stream towards the dentate gyrus had become obvious, and the labeled cells in the dentate gyrus began to form a C-shaped layer structure. The radial migration of the labeled cells from the ventricular germinative zone facing the Ammon's horn also became evident at this stage.
The migratory cells were widely distributed throughout the entire Ammon's horn and reached the forming stratum lacunosum/moleculare through the pyramidal cell layer.

\section{Transition of TN-C-positive cells to GFAP- immunoreactive astrocytes}

At P2, TN-C-positive cells in the dentate gyrus were stratified along the layer of the $\mathrm{C}$-shaped granule cell layer (Fig. 2A). The somata of the TN-C-positive 
cells aligned in the dentate gyrus extended GFAP-immunoreactive radial processes towards the pia mater, as demonstrated by the double labeling with TN-C in situ hybridization and GFAP-immunostaining (Fig. 2B). The morphology of these labeled cells was reminiscent of the specialized unipolar astrocytes found in the dentate gyrus at the adult stage (LUDWIN et al., 1976; KOSAKA and HAMA, 1986). Small numbers of double labeled cells with a stellate morphology were noted in the dentate hilar region (Fig. 2B). The numbers of TN-C-positive cells in the ventricular germinative zone had diminished significantly at this stage (Fig. 2A).

The TN-C-positive cells in the Ammon's horn were distributed mainly in the stratum lacunosum/ moleculare, with small numbers of them also being found within the pyramidal cell layer (Fig. 2A). The TN-C-positive cells in the stratum lacunosum/ moleculare extended GFAP-immunoreactive processes and displayed a stellate morphology (Fig. 2C). In the pyramidal cell layer, GFAP-immunoreactive radial glial processes traversed the cortex, and TN-Cpositive cells that were closely apposed to these processes were occasionally found (Fig. 2D).

\section{Interrelations between migratory astroglial precursors and radial glial arrangement}

At E17, TN-C-positive cells had migrated from the ventricular germinative zone towards the dentate gyrus, and GFAP-immunopositive radial glial processes had extended from the restricted area of the ventricular germinative zone closely apposed to the forming fimbria (Fig. 3A). The radial glial processes extended through the boundary between the fimbria and the Ammon's horn and approached the forming dentate gyrus through the subpial parenchyma (Fig. $3 A)$.

The origin and arrangement of the GFAP-immunopositive processes coincided with the origin and migratory route of the TN-C-positive cells in the direction of the dentate gyrus. These cells, which appeared to migrate from the ventricular germinative zone, were closely apposed to the GFAP-immunoreactive radial glial processes (Fig. 3B). A close apposition between the GFAP-immunopositive radial processes and the TN-C-positive cells was also noted in the subpial area on the migratory route leading to the dentate gyrus (Fig. 3C).

The migration of large numbers of TN-C-positive cells was observed in the Ammon's horn at E18 (Fig. 1D). Several TN-C-positive cells in the pyramidal cell layer were also apposed to the GFAP-immunoreactive radial glial processes that traversed the
Ammon's horn in the neonatal hippocampus (Fig. 2D).

\section{DNA-replicating activity of TN-C-positive cells}

The DNA-replicating activity of TN-C-positive cells was examined in the neonatal hippocampus. Double labeling by in situ hybridization for TN-C mRNA and by immunohistochemical detection of incorporated $\mathrm{BrdU}$ was performed at $\mathrm{P} 0$. Stratified TN-Cpositive cells were found in the forming dentate gyrus (Fig. 4A). Some of these cells incorporated BrdU, as observed at a higher magnification of the suprapyramidal blade (Fig. 4B). The BrdU-positive TN-Cnegative cells that were occasionally found were presumed to be granule cells in the S-phase. Double labeled cells were also detected in the forming stratum oriens, where the migration of TN-C-positive cells towards the dentate gyrus was also still observed (Fig. 4C). BrdU-positive TN-C-negative cells were also present in the stream and were considered to be migratory dentate granule cells in the S-phase.

Double labeled cells were also present in the ventricular germinative zone facing the entire Ammon's horn. Their migration towards the cortex was still evident (Fig. 4D).

\section{DISCUSSION}

The development of astrocytes in the embryonic hippocampus of the hamster has been studied using GFAP as a marker because GFAP expression occurs at an early stage of development in this species (SIEvERS et al., 1992). Unlike the hamster, other rodents express GFAP-immunoreactivity at a much later stage in hippocampal astroglial development. Consequently, little has been known about astrogliogenesis in the mouse embryonic hippocampus. An early marker for mouse astroglial precursors was thus needed.

In situ hybridization histochemistry for TN-C mRNA has been successfully applied as an early marker in the investigation of astrogliogenesis in the mouse embryonic cerebrum (YUASA, 2001). Using this marker, the present study demonstrates the mode of generation, migration, and settlement of astroglial precursors in the developing mouse hippocampus. The colocalization of GFAP-immunoreactivity in TN-C. positive cells during the early postnatal stage and TN-C gene expression in the ventricular germinative zone of the embryo indicate that $\mathrm{TN}$-C gene expression can be used as an early marker for mouse hippocampal astroglial precursors prior to the expression of GFAP. The proposed developmental 
A
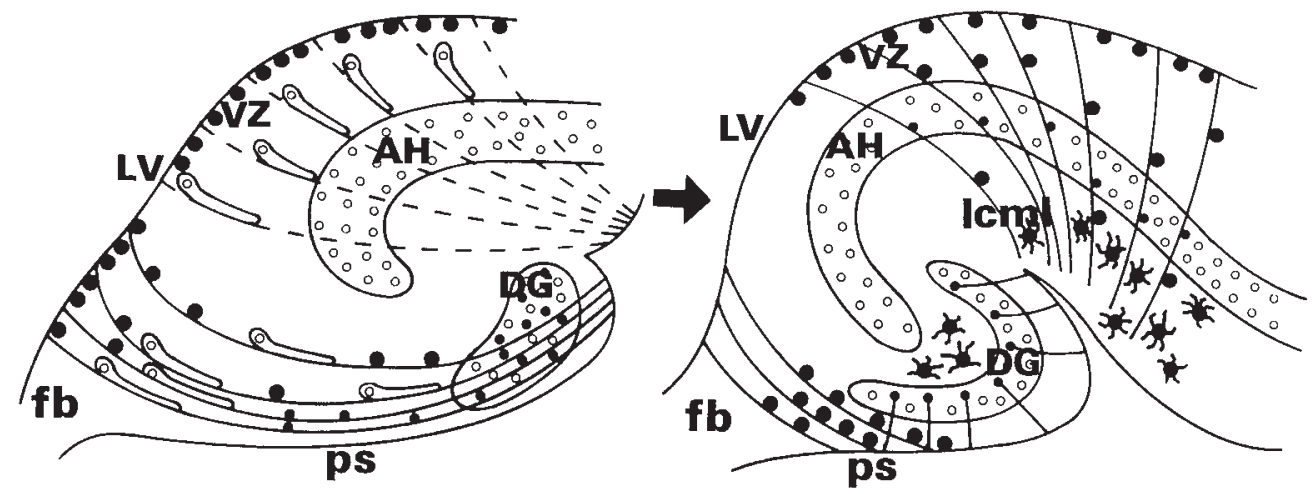

Fig. 5. Schematic drawings of the migration, settlement and differentiation of TN-C-positive cells (astroglial precursors) in the developing hippocampus. A. Embryonic stage around E15-17. GFAP-positive radial glia extend from a restricted area of the ventricular germinative zone apposed to the fimbria. The migration of dentate granule cells is guided by contact with these processes. TN-C-positive cells are generated from the ventricular germinative zone closely apposed to the fimbria and migrate along the GFAP-positive processes to accumulate in the forming dentate gyrus. In contrast, radial glial processes extending from a wide area of the ventricular germinative zone and traversing the Ammon's horn display no GFAP-immunoreactivity but do exhibit vimentin-immunoreactivity (RICKMANN et al., 1987). Radial migration of the pyramidal neurons is guided by contact with these processes. B. Perinatal stage around E18-P2. TN-C-positive cells are aligned in the dentate gyrus and extend GFAP-positive processes towards the pia mater. TN-C-positive cells are still present in the subpial area on the migratory route. Radial glial processes in the Ammon's horn exhibit GFAPimmunoreactivity. Radial migration of TN-C-positive cells along these processes becomes evident after E18. The labeled cells in the stratum lacunosum/moleculare extend GFAP-positive processes and display a stellate morphology. Solid lines on the radial glia indicate GFAP-positive processes; broken lines indicate GFAPnegative, vimentin-positive processes. Bright elongated cells represent migratory neurons; dark round cells represent TN-C-positive cells. Stellate cells indicate differentiated astrocytes that express both $T N$-C and GFAP.

processes are illustrated schematically in Figure 5.

The above-mentioned study using hamsters showed that GFAP-positive astrocytes in the embryonic dentate gyrus are generated from the subpial dentate matrix, which is situated between the fimbria and the forming dentate gyrus (SIEvers et al., 1992). The subpial matrix is thought to be formed by astroglial precursors derived from the ventricular germinative zone apposed to the fimbria. The precursors then migrate through the subpial area to the dentate gyrus.

The present study on the mouse hippocampus demonstrates that TN-C-positive cells are generated in the ventricular germinative zone near the fimbria and then migrate through the subpial area towards the dentate gyrus. The migration of these labeled cells begins at E15 and persists until the early postnatal stage (Figs. 1-4). TN-C-positive cells during migration or shortly after their arrival at the dentate gyrus do not display GFAP-immunoreactivity, as observed in E17 specimens (Fig. 3A). TN-C-positive cells aligned in the dentate gyrus extend GFAP-immunopositive radial processes towards the pia mater at P2 (Fig. 2B), indicating that the TN-C-positive cells are the precursors of dentate unipolar astrocytes. The differentiation of these precursors to unipolar astrocytes is therefore considered to occur after their final settlement in the dentate gyrus. These TN-C gene expression findings in the mouse confirm the origin and migratory route of astroglial precursors as revealed by GFAP in the hamster dentate gyrus (SIEVERS et al., 1992).

TN-C-positive cells along the subpial migratory route showed a DNA-replicating activity (Fig. 4C). These double-labeled cells should thus continuously supply the astroglial precursors in the direction of the dentate gyrus. This finding confirms the significance of the subpial dentate matrix, as shown in the hamster hippocampus (SiEvers et al., 1992). TN-Cpositive cells aligned in the dentate gyrus also demon- 
strated a DNA-replicating activity during the early postnatal stage (Fig. 4B). These double-labeled cells are considered one of the origins of astrocytes in the area surrounding the dentate gyrus during the postnatal stage.

The previous study on TN-C-positive astroglial precursors in the mouse embryonic cerebrum (YUASA, 2001) demonstrated that the ventricular germinative zone was differentiated in a mosaic pattern. The present study revealed that TN-Cpositive cells in the dentate gyrus and those in the Ammon's horn have different origins (both time and site) and different modes of migration. The precursors of dentate astrocytes originated from a restricted area of the ventricular germinative zone apposed to the fimbria and migrated through the subpial parenchyma. This migratory pattern coincides well with that of the dentate granule cells, as described in the mouse (SMART, 1982; REZNIKOV, 1991) and rat (SCHLE. SSINGER et al., 1975; RICKMANN et al., 1987; ALTMAN and BAYER, 1990a, c). In contrast, the astroglial precursors for the Ammon's horn originated from a wide area of the ventricular germinative zone and migrated radially towards the cortex. This pattern also coincides well with that of Ammon's horn pyramidal neurons, as described in the mouse (SMART, 1982; REZNIKOV, 1991), rat (ALTMAN and BAYER, 1990b) and rhesus monkey (NOWAKOWSKI and RAKIC, 1979). These findings suggest that neurogenesis and astrogliogenesis in the hippocampus exhibit common characteristics with regard to the mosaic organization of the ventricular germinative zone and cell migration from this location.

Shared mechanisms might also be involved in the guidance of the migration of both neurons and astrocytes in the developing hippocampus. Directional neuronal migration is thought to be guided by contact with radial glial processes (RAKIC, 1990). Migratory dentate granule cells have been shown to be guided by radial glial processes extending from the ventricular germinative zone apposed to the fimbria leading to the forming dentate gyrus (RICKMANN et al., 1987). As shown in the present study, the arrangement of GFAP-immunoreactive radial glial processes extending from the restricted ventricular germinative zone coincides well with the migratory route of TN-C-positive cells in the embryonic dentate gyrus (Fig. 3). The migration of glial precursors during the postnatal stage has been suggested to be guided by contact with radial glial processes in common with migratory neurons (LEVISON et al., 1993; LEVISON and GOLDMAN, 1993; NEWMAN et al., 1995; ZERLIN et al., 1995). These findings suggest that unipolar astroglia in the dentate gyrus are generated from the same site and migrate on the same pathway as the dentate granule cells.

Contact guidance of migratory pyramidal cells by radial glial processes has been reported in the embryonic Ammon's horn (NowAKOWSKI and RAKIC, 1979). The apposition of TN-C-positive cells and GFAP-immunoreactive radial processes was also noted in the mouse neonatal Ammon's horn, as shown in Figure 2D. These observations suggest that astroglial precursors in the Ammon's horn may be generated and migrate in the same manner as pyramidal cells. The present findings suggest that the migration of astroglial precursors in the embryonic and neonatal hippocampus is guided by the radial glial processes that are involved in neuronal migration.

Other possible origins of astrocytes in the hippocampus should be considered. The transformation of GFAP-immunoreactive radial glia extending from the ventricular germinative zone may represent one of the origins of hippocampal astroglial subpopulations, as proposed for the developing neocortex (CHOI and LAPHAM, 1978; CuliCAN et al., 1990; EDWARDS et al., 1990; Hunter and HAtTEN, 1995; MARINPADILLA, 1995). These findings suggest that hippocampal astrocytes have heterogeneous origins that depend on their localization and morphology. The quantitative contribution of each origin to the hippocampal astrocyte population needs to be investigated. Functional heterogeneity in astroglial subsets has been well recognized (WILKIN et al., 1990), but the interrelations between the heterogeneous origins and functional differences in each subset of hippocampal astrocytes will have to be determined in future studies.

Acknowledgments. The author is indebted to Prof. K. IKENAKA (National Institute for Physiological Sciences) for invaluable discussions.

\section{REFERENCES}

Altman, J. and S. A. BAYER: Mosaic organization of the hippocampal neuroepithelium and the multiple germinal sources of dentate granule cells. J. Comp. Neurol. 301: 325-342 (1990a).

- Prolonged sojourn of developing pyramidal cells in the intermediate zone of the hippocampus and their settling in the stratum pyramidale. J. Comp. Neurol. 301: 343-364 (1990b).

- Migration and distribution of two populations of hippocampal granule cell precursors during the perinatal and postnatal periods. J. Comp. Neurol. 301: 365-381 (1990c). 
Bartsch, S., U. Bartsch, U. Doerries, A. Faissner, A. Weller, P. Ekblom and M. Schachner: Expression of tenascin in the developing and adult cerebellar cortex. J. Neurosci. 12: 736-749 (1992).

Chor, B. H. and L. W. LAPHAM : Radial glia in the human fetal cerebrum: a combined Golgi, immunofluorescent and electron microscopic study. Brain Res. 148: 295-311 (1978).

Culican, S. M., N. Baumrind, M. Yamamoto and A. L. Pearlman: Cortical radial glia: identification in tissue culture and evidence for their transformation to astrocytes. J. Neurosci. 10: 684-692 (1990).

Doerries, U. and M. SChachner: Tenascin mRNA isoforms in the developing mouse brain. J. Neurosci. Res. 37: 336-347 (1994).

Edwards, M. A., M. Yamamoto and V. S. Caviness, Jr: Organization of radial glia and related cells in the developing murine CNS: an analysis based upon a new monoclonal antibody marker. Neuroscience 36 : 121-144 (1990).

Hunter, K. E. and M. E. HATten: Radial glial cell transformation to astrocytes is bidirectional: regulation by a diffusible factor in embryonic forebrain. Proc. Nat. Acad. Sci. USA 92: 2061-2065 (1995).

Kosaka, T. and K. HAMA : Three-dimensional structure of astrocytes in the rat dentate gyrus. J. Comp. Neurol. 249: 242-260 (1986).

Levison, S. W. and J. E. GoLdMaN: Both oligodendrocytes and astrocytes develop from progenitors in the subventricular zone of postnatal rat forebrain. Neuron 10: 201-212 (1993).

Levison, S. W., C. Chuang, B. J. Abramson and J. E. GoLDMAN: The migration patterns and developmental fates of glial precursors in the rat subventricular zone are temporally regulated. Development 119: 611-622 (1993).

Ludwin, S. K., J. C. KoseK and L. F. ENG: The topographical distribution of S100 and GFAP proteins in the adult rat brain: an immunohistochemical study using horseradish peroxidase-labelled antibodies. J. Comp. Neurol. 165: 197-208 (1976).

Marin-Padilla, M.: Prenatal development of fibrous (white matter), protoplasmic (gray matter), and layer I astrocytes in the human cerebral cortex: a Golgi study. J. Comp. Neurol. 357: 554-572 (1995).

Mitrovic, M., U. Doerries and M. SChachner: Expression of the extracellular matrix glycoprotein tenascin in the somatosensory cortex of the mouse during postnatal development: an immunocytochemical and in situ hybridization analysis. J. Neurocytol. 23: 364-378 (1994).

Newman, S. R., M. ZerLin and J. E. Goldman: Glial progenitors from the postnatal subventricular zone migrate along radial glia in vitro. Abst. Soc. Neurosci. 414. 12 (1995)

Nowakowski, R. S. and P. RAKIC: The mode of migration of neurons to the hippocampus: a Golgi and electron microscopic analysis in foetal rhesus monkey. J. Neurocytol. 8: 697-718 (1979).
RAKIC, P.: Principles of neural cell migration. Experientia 46: 882-891 (1990).

Reznikov, K. Y.: Cell proliferation and cytogenesis in the mouse hippocampus. Adv. Anat. Embryol. Cell Biol. 122: 1-83 (1991).

Rickmann, M., D. G. Amaral and W. M. Cowan: Organization of radial glial cells during the development of the rat dentate gyrus. J. Comp. Neurol. 264: 449-479 (1987).

Saga, Y., T. Tsukamoto, N. Jing, M. Kusakabe and T. SakakURA: Murine tenascin: cDNA cloning, structure and temporal expression of isoforms. Gene 104: 177-185 (1991).

Schlessinger, A. R., W. M. Cowan and D. I. Gottlieb: An autoradiographic study of the time of origin and the pattern of granule cell migration in the dentate gyrus of the rat. J. Comp. Neurol. 159: 149-176 (1975).

Sievers, J., D. Hartmann, F. W. Pehlemann and M. BERRY: Development of astroglial cells in the proliferative matrices, the granule cell layer, and the hippocampal fissure of the hamster dentate gyrus. J. Comp. Neurol. 320: 1-32 (1992).

SMart, I. H. M.: Radial unit analysis of hippocampal histogenesis in the mouse. J. Anat. 135: 763-793 (1982).

Wilkin, G. P., D. R. Marriot and A. J. Cholewinski: Astrocyte heterogeneity. TINS 13: 43-46 (1990).

YuAsa, S.: Bergmann glial development in the mouse cerebellum as revealed by tenascin expression. Anat. Embryol. 194: 223-234 (1996).

- Development of astrocytes in the mouse embry onic cerebrum tracked by tenascin-C gene expression. Arch. Histol. Cytol. 64: 119-126 (2001).

Zerlin, M., S. W. Levison and J. E. Goldman: Early patterns of migration, morphogenesis, and intermediate filament expression of subventricular zone cells in the postnatal rat forebrain. J. Neurosci. 15: 7238-7249 (1995).
Prof. Shigeki YUASA

Department of Anatomy

and Developmental Biology

Chiba University School of Medicine

Chiba, 260-8670 Japan

Fax: +81-43-226-2021

E-mail: yuasa@med.m.chiba-u.ac.jp

湯浅茂樹 260-8670 千葉市中央区亥鼻 1-8-1

千葉大学医学部

解剖学第二講座 